\title{
Treatment Decision-making of Cracked Teeth: Survey of American Endodontists
}

\author{
Ayman M Abulhamael ${ }^{1,2}$, Radhika Tandon ${ }^{3}$, Ziyad T Alzamzami ${ }^{4}$, Loai Alsofi ${ }^{5}$, Rafael A Roges ${ }^{6}$, Ilan Rotstein ${ }^{7}$
}

\begin{abstract}
Aim: The aim of this study is to survey the opinion of endodontists in the United States regarding their approach to treatment of cracked teeth. Materials and methods: A survey assessing the opinions of 3,500 endodontists in the United States was administered over a 4-week period. It consisted of a hypothetical clinical case and eight different treatment scenarios. Participants were asked to select one of the two treatment options: (A) informing the patient of the presence of a crack, reinforcing the tooth, and continuing with endodontic treatment; and (B) extraction of the tooth followed by replacement with an implant supported crown or fixed partial denture.

Results: When the crack extended across the distal marginal ridge with no associated probing depths, $92.65 \%$ endodontists preferred to continue with endodontic treatment and reenforcing the tooth. When the crack extended across the distal marginal ridge and was associated with a probing depth of $6 \mathrm{~mm}, 80 \%$ preferred extraction of the tooth. When the crack extended across the mesial marginal ridge up to the orifice of the mesiolingual canal with no associated probing depth, $82.78 \%$ preferred to continue with the treatment. When the crack was associated with a $6-\mathrm{mm}$ probing depth, $83.79 \%$ preferred tooth extraction. When the crack extended across the mesial marginal ridge and down into the mesiolingual canal with/without associated probing depth, $91.13 \%$ and $63.54 \%$ preferred tooth extraction, respectively. When the crack involved both mesial and distal marginal ridges and extended across the pulp chamber, $79.74 \%$ preferred tooth extraction. When a split tooth was presented, $98.48 \%$ preferred tooth extraction.

Conclusion: It appears that the presence of a 6- $\mathrm{mm}$ periodontal pocket is considered an important factor by most American endodontists when deciding whether to preserve the cracked tooth or extract it.

Clinical significance: There is no consensus among dentists regarding the best approach to treat cracked teeth. Conclusive studies evaluating clinical approaches of endodontists regarding treatment of cracked teeth are lacking. Therefore, surveyed opinion of endodontists in the United States regarding their approach to treatment of cracked teeth was done to try to reach the best clinical decision regarding this dilemma. Keywords: Cracked tooth, Probing depth, Root canal treatment, Tooth survival.

The Journal of Contemporary Dental Practice (2019): 10.5005/jp-journals-10024-2554
\end{abstract}

\section{INTRODUCTION}

Cracked teeth can present a clinical challenge due to their complex diagnosis and unpredictable prognosis. Treatment often varies depending on the nature of the crack and clinical experience of the operator. In 1964, Cameron" coined the term "cracked tooth syndrome" for teeth that exhibited discomfort upon biting and thermal sensitivity. Rivera and Walton ${ }^{2}$ defined a cracked tooth as an incomplete fracture initiating from the crown and extending subgingivally, usually in a mesiodistal direction. The crack may extend through one or both of the marginal ridges and through the proximal surfaces. It can be limited to the crown portion of the tooth or extend further to the root. ${ }^{2}$ Five types of cracks were described: craze lines, fractured cusps, cracked tooth, split tooth, and vertical root fracture. ${ }^{2}$

Cracked teeth are difficult to diagnose clinically because of the complicated and diverse symptoms associated with this condition and the challenge in locating crack lines. According to Abbott and Leow, $^{3}$ it is imperative to remove all restorations prior to endodontic treatment. Once the restoration is removed, the mesial and distal marginal ridges can be examined with a combination of dyes, transillumination, and the operating microscope. In some cases, diagnostic exploratory surgery is necessary to rule out the presence of a crack. ${ }^{3}$

Depending on the apical extension of the crack, periodontal probing depths may vary. ${ }^{4-7}$ Berman and Kuttler $^{8}$ described fracture necrosis in teeth that did not have any restorations. They attributed
${ }^{1}$ Department of Endodontics, Faculty of Dentistry, King Abdulaziz University, Jeddah, Saudi Arabia

${ }^{2}$ Clinical Dentistry of Division of Endodontics, Orthodontics and General Practice Residency in the Herman Ostrow School of Dentistry of University of Southern California, Los Angeles, California, USA

${ }^{3}$ Private practice San Francisco and the Bay Area

${ }^{4,5}$ Department of Endodontics, Faculty of Dentistry, King Abdulaziz University Dental Hospital, Jeddah, Saudi Arabia

${ }^{6,7}$ Department of Endodontics at the Herman Ostrow School of Dentistry of the University of Southern California, Los Angeles, California, USA

Corresponding Author: Ayman M Abulhamael, Department of Endodontics, Faculty of Dentistry, King Abdulaziz University, Jeddah, Saudi Arabia, Phone: +966 598977241; e-mail: ayman.abulhamael@ hotmail.co.uk

How to cite this article: Abulhamael AM, Tandon R, et al. Treatment Decision-making of Cracked Teeth: Survey of American Endodontists. J Contemp Dent Pract 2019;20(5):543-547.

Source of support: Nil

Conflict of interest: None

it to cracks that traveled from the occlusal surface and involved the pulp of the affected teeth. Based on their findings, they recommended extraction of the affected teeth as a primary treatment option due to poor prognosis of endodontic treatment in such cases. ${ }^{8}$ 


\section{Table 1: The Jowa staging index}

Stage I: No probing pocket depths $>5 \mathrm{~mm}$ and no crack across the distal marginal ridge

Stage II: No probing pocket depths $>5 \mathrm{~mm}$, having a crack across the distal marginal ridge, and not having a periapical diagnosis of CAP/SAP/AAA

Stage III: No probing pocket depths $>5 \mathrm{~mm}$, having a crack across the distal marginal ridge, and having a periapical diagnosis of CAP/ SAP/AAA

Stage IV: One mesial or distal probing pocket depth $>5 \mathrm{~mm}$

CAP, Chronic apical periodontitis; SAP, symptomatic apical periodontitis; AAA, acute apical abscess

According to Eakle et al., ${ }^{9} 48 \%$ of cracked teeth are mandibular molars. Kang et al., ${ }^{10}$ reported similar findings. The mandibular second molar showed the highest incidence of cracks. ${ }^{10}$ In addition, intact teeth and teeth with class I occlusal cavities exhibited a higher incidence of cracks., ${ }^{9,10}$

Most outcome studies on cracked teeth used survival as a metric. Tan et al., ${ }^{11}$ reported $85 \%$ survival rate of cracked teeth 2 years after completion of root canal treatment. Kang et al., ${ }^{10}$ found the 2-year survival rate of cracked teeth to be influenced mostly by the presence of probing depth of $6 \mathrm{~mm}$ or more. Teeth with a probing depth greater than $6 \mathrm{~mm}$ had a survival rate of $74 \%$ whereas those with probing depth less than $6 \mathrm{~mm}$ had a survival rate of $96 \%$. Extension of the crack into the pulpal floor resulted in a significant increase in tooth loss. ${ }^{12}$ The survival rate of cracked teeth treated endodontically and restored with crowns (95.1\%) was higher than those treated endodontically and restored with composite resin $(77 \%)^{13}$
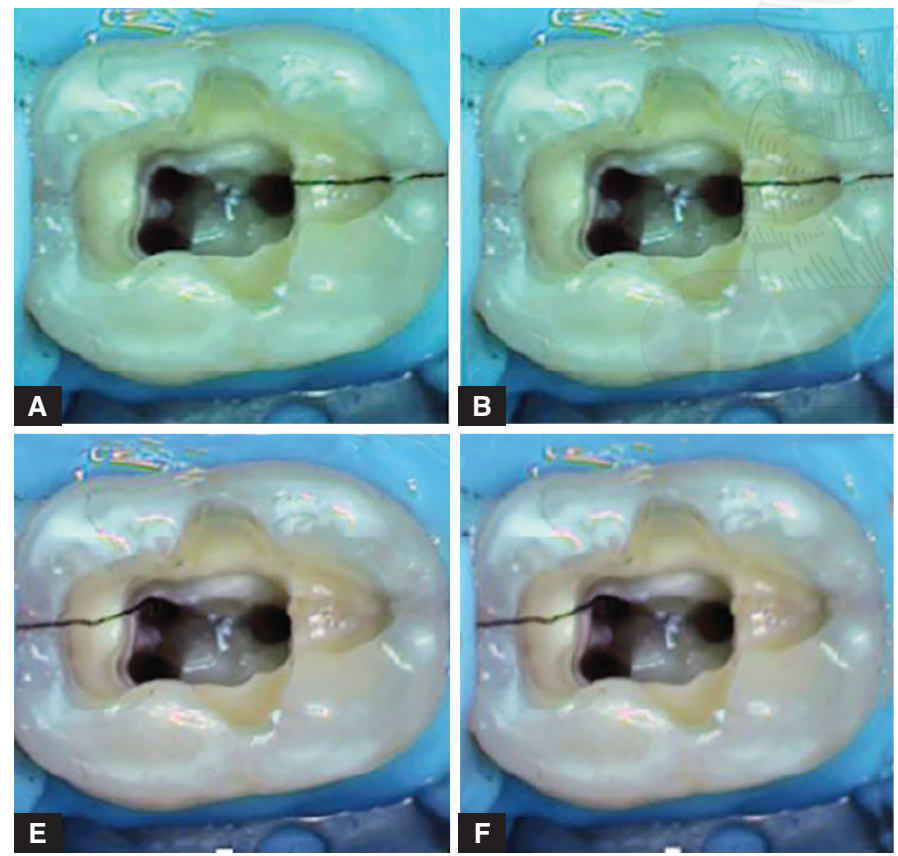

Recently, Krell and Caplan ${ }^{14}$ described the outcome of cracked teeth based on a healing criterion rather than survival. They defined success as "absence of signs and symptoms plus resolution of any previous pathoses." Three diagnostic factors were suggested: (1) presence of one probing pocket depth $>5 \mathrm{~mm}$; (2) presence of a crack across the distal marginal ridge; and (3) periapical diagnosis of chronic apical abscess, symptomatic apical periodontitis, or acute apical abscess. ${ }^{14}$ Based on their findings, the lowa stage index for crack teeth was suggested (Table 1). This index was designed to aid in clinical treatment decision-making.

There is no consensus among endodontists regarding the best approach to treat cracked teeth. Conclusive studies evaluating clinical approaches of endodontists regarding treatment of cracked teeth are lacking. The aim of this study is, therefore, to survey the opinion of endodontists in the United States regarding their approach to the treatment of cracked teeth.

\section{Materials and Methods}

An invitation to participate in an online survey administered through Google docs was e-mailed to 3500 US endodontists listed as "active" in the online membership directory of the American Association of Endodontists (AAE). The survey was administered over a 4-week period between April 2018 and May 2018 in the United States. It consisted of a description of a hypothetical clinical case (Box 1) and eight different scenarios of clinical presentation and treatment of cracked teeth (Fig. 1). Each scenario was described along with a corresponding clinical image. Participants were asked to select one of the two treatment options as follows: $(A)$ informing the patient of the presence of a crack, reinforcing the tooth,

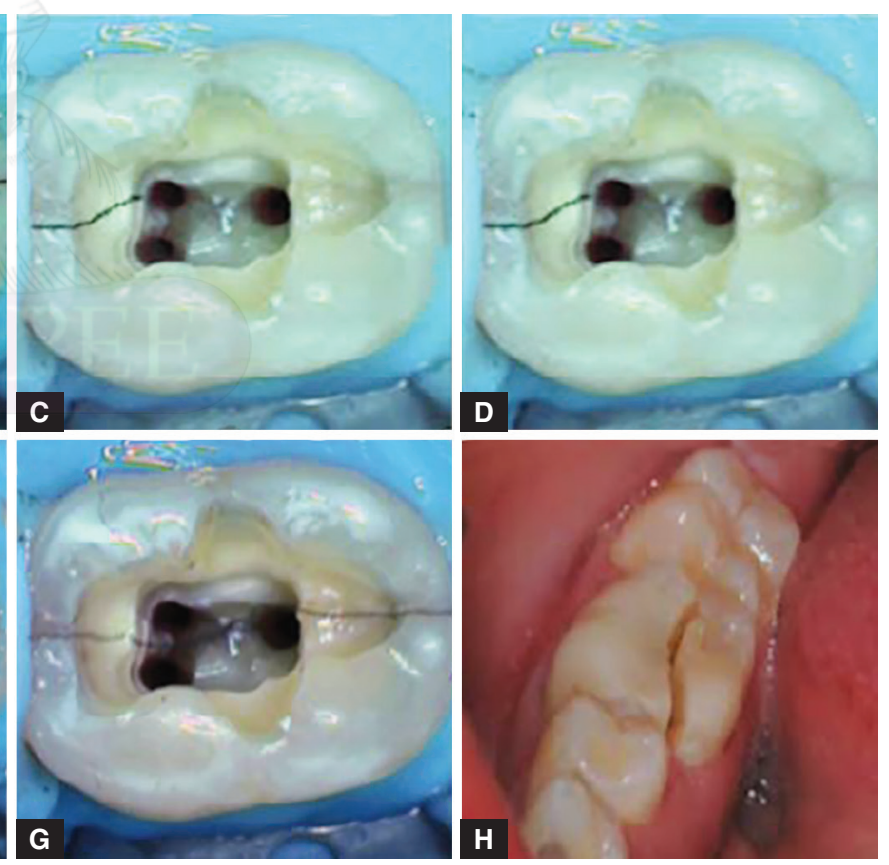

Figs 1 A to H:The clinical scenarios presented to survey participants. (A) A crack line across the distal marginal ridge with no associated probing depth. (B) A crack line across the distal marginal ridge with a 6-mm probing depth on the distal aspect of the root associated with the crack line. (C) A crack line across the mesial marginal ridge extending up to the orifice of the mesiolingual canal with no associated probing depth. (D) A crack line across the mesial marginal ridge extending up to the orifice of the mesiolingual canal with a 6-mm probing depth on the mesial aspect of the root associated with the crack line. (E) A crack line across the mesial marginal ridge extending down the mesiolingual canal with no associated probing depth. (F) A crack line across the mesial marginal ridge extending down the mesiolingual canal with a 6-mm probing depth on the mesial aspect of the root associated with the crack line. (G) A crack line across the mesial marginal ridge extending across the pulp chamber involving the distal marginal ridge with no associated probing depth. $(\mathrm{H}) \mathrm{A}$ crack extending in the mesiodistal aspect of the tooth splitting it into two segments 


\section{Box 1}

Case description

A middle-aged patient presents to your endodontic office for evaluation and treatment of the mandibular first molar. Upon examination, you established a pulpal diagnosis of necrotic pulp and a periapical diagnosis of symptomatic apical periodontitis. What would be your treatment if after removing all restorations and creating the access preparation the following is found: Figure $1(\mathrm{~A}-\mathrm{H})$

and continuing with endodontic treatment; and (B) extraction of the tooth followed by replacement with an implant supported crown or fixed partial denture. To encourage respondents to answer all questions, an error message was delivered if questions were left unanswered. Participants were informed that the survey was a research project and that anonymity of participants was assured.

\section{RESULTS}

Three hundred and ninety-five endodontists responded to the survey, representing an $11.28 \%$ response rate. About $63.29 \%$ of participants were in practice for more than 10 years. About $22.78 \%$ were in practice for 5 to 10 years and the remaining 13.92\% have been in practice for less than 4 years (Graph 1 and Table 2).

In scenario 1, where the crack extended across the distal marginal ridge with no associated probing depths, $92.65 \%$ of participants answered that they would continue with endodontic treatment after informing the patient of the presence of the crack and reenforcing the tooth (Graph 2 and Table 2). In scenario 2, where the crack extended across the distal marginal ridge and

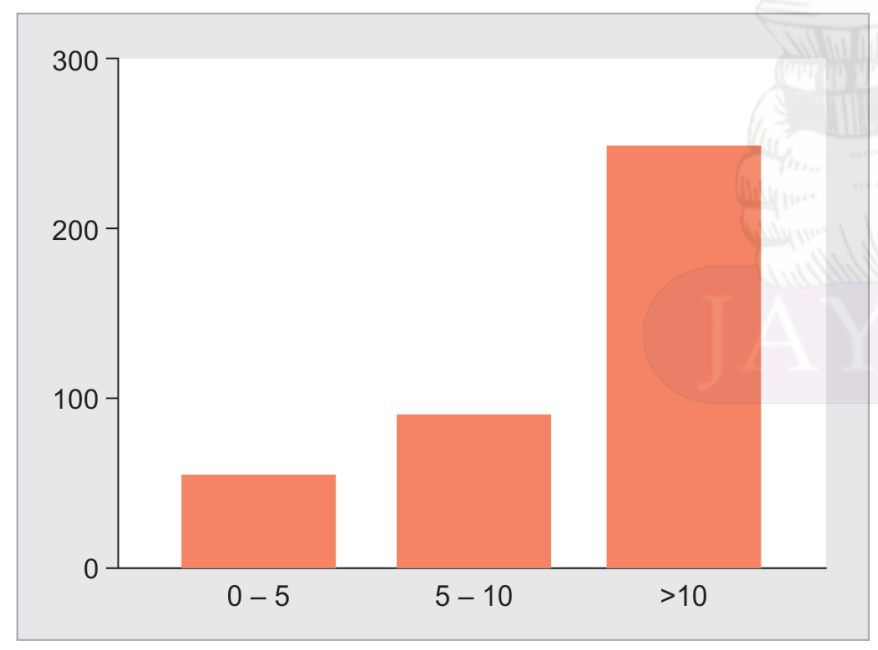

Graph 1: Number of years in practice of survey participants $(n=395)$ was associated with a probing depth of $6 \mathrm{~mm}, 80 \%$ of participants chose tooth extraction (Graph 2 and Table 2).

In scenario 3, where the crack line extended across the mesial marginal ridge up to the orifice of the mesiolingual canal with no associated probing depth, $82.78 \%$ of respondents chose to continue with the treatment after informing the patient (Graph 3 and Table 2).

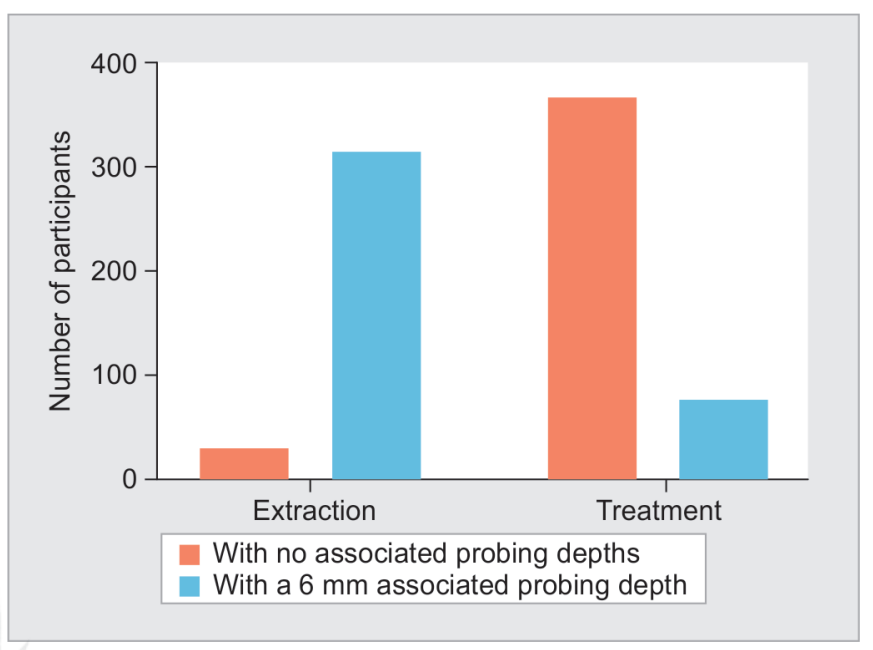

Graph 2: Opinion distribution regarding treatment of a crack line across the distal marginal ridge with no associated probing depth $(n=366)$ and with a $6 \mathrm{~mm}$ associated probing depth $(n=79)$

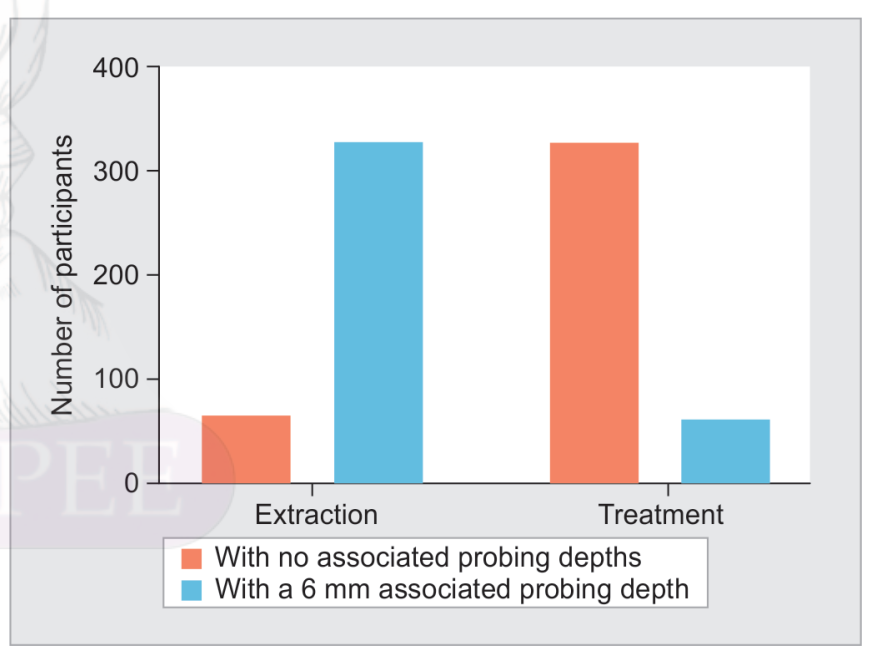

Graph 3: Opinion distribution regarding treatment of a crack line across the mesial marginal ridge extending up to the orifice of the mesiolingual canal with no associated probing depth $(n=327)$ and with a $6-\mathrm{mm}$ probing depth on the mesial aspect of the root associated with the crack line $(n=64)$

Table 2: Summary of opinion distributions regarding crack lines

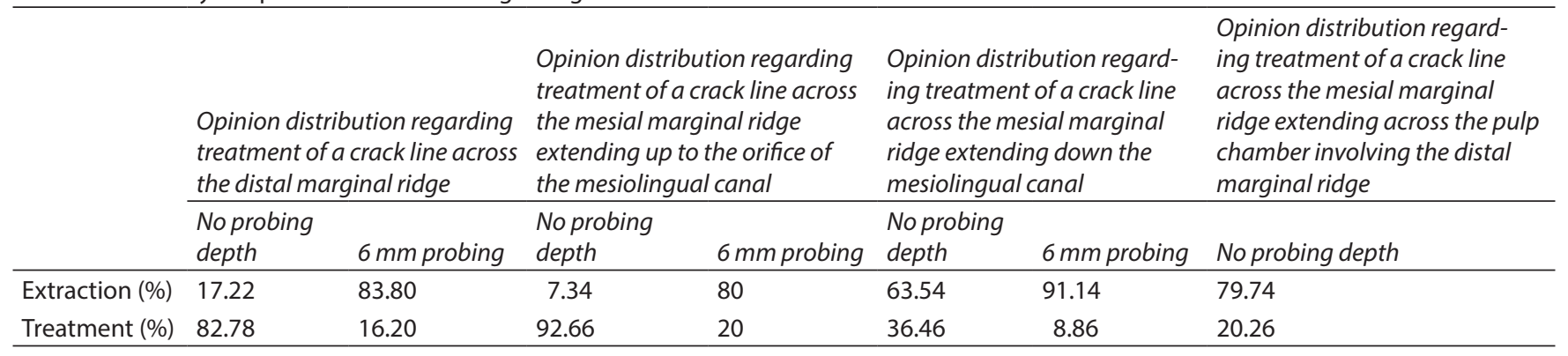




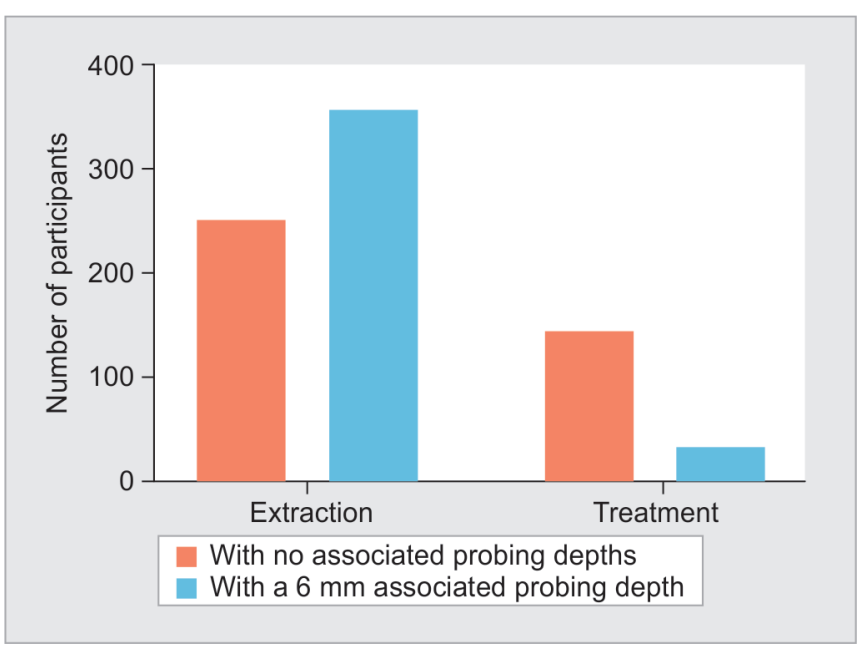

Graph 4: Opinion distribution regarding treatment of a crack line across the mesial marginal ridge extending down the mesiolingual canal with no associated probing depth $(n=144)$ and with a $6-\mathrm{mm}$ probing depth on the mesial aspect of the root associated with the crack line $(n=35)$

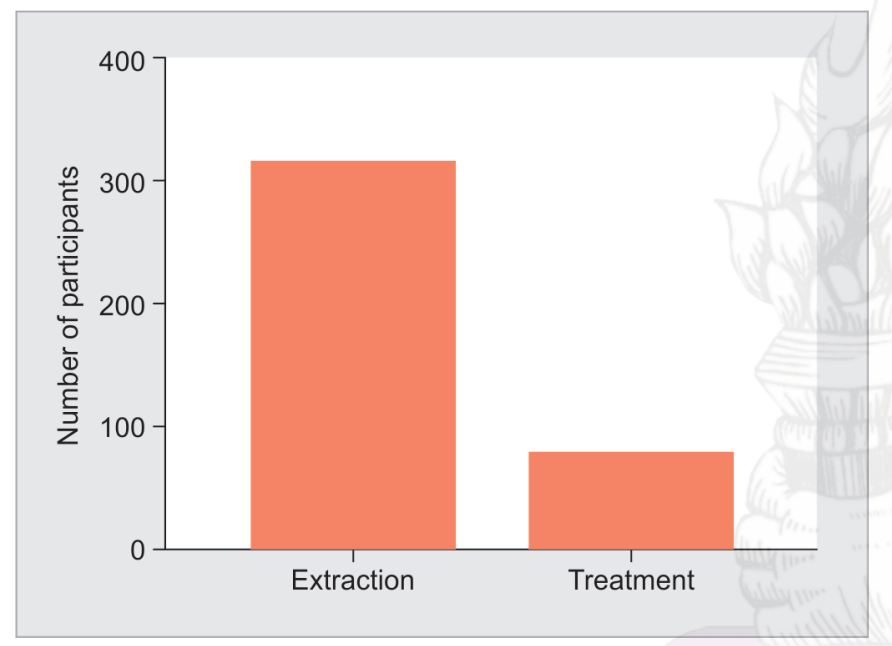

Graph 5: Opinion distribution regarding treatment of a crack line across the mesial marginal ridge extending across the pulp chamber involving the distal marginal ridge with no associated probing depth $(n=80)$

In scenario 4, where the crack was associated with a 6-mm probing depth, $83.79 \%$ of respondents chose tooth extraction (Graph 3 and Table 2). In scenario 5, where the crack extended across the mesial marginal ridge and down into the mesiolingual canal without associated probing depth, $63.54 \%$ of respondents chose tooth extraction (Graph 4 and Table 2). When the same scenario was described but with an associated probing depth, $91.13 \%$ of participants chose tooth extraction (Graph 4 and Table 2).

When the crack line involved both mesial and distal marginal ridges and extended across the pulp chamber, $79.74 \%$ of participants chose tooth extraction (Graph 5 and Table 2). When a scenario of a split tooth was presented, $98.48 \%$ of respondents chose tooth extraction (Graph 6).

\section{Discussion}

When presented with a cracked tooth, clinicians face a dilemma of whether to treat it endodontically or to extract it. The results of the present study support the findings of Krell and Caplan ${ }^{14}$ indicating

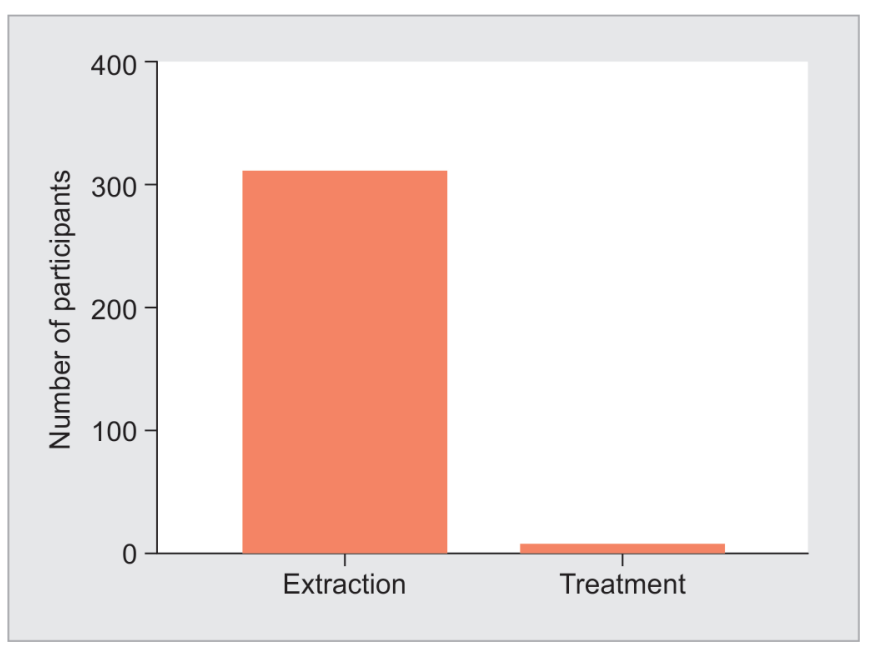

Graph 6: Opinion distribution regarding treatment of a crack extending in the mesiodistal aspect of the tooth splitting it into two segments $(n=6)$

that the presence of a probing depth of $5 \mathrm{~mm}$ or more is a significant factor impacting the prognosis and long-term survival of the tooth. Our results also agree with those of Kang et al., ${ }^{10}$ who reported that 2-year survival rate of root-filled cracked teeth with a probing depth of more than $6 \mathrm{~mm}$ was significantly lower than that of teeth with probing depths of less than $6 \mathrm{~mm}$.

In the scenario where the crack was crossing the pulpal floor, most participants preferred extraction of the tooth. This further supports the results of Sim et al., ${ }^{12}$ who reported that extension of cracks onto the pulpal floor independently increased the odds of tooth loss by 11-fold. However, the 5-year survival rate when the crack did not extend into the pulpal floor was 99\%. On the contrary, the extension of the crack into the pulpal floor lowered the 5 -year survival rate to $88 \%$. Early diagnosis of the crack and a timely reinforcement of the tooth will yield a better long-term prognosis. $3,15,16$

In our study, a clear trend was found when endodontists were asked about treating cracked teeth with associated periodontal probing depths. The majority of respondents thought that extraction was the treatment of choice. Also, in the case of a split tooth, the vast majority of respondents thought that extraction was the treatment of choice. Nevertheless, $6 \%$ of endodontists preferred to try and save the tooth by performing endodontic treatment and coronal restoration. It can be presumed that these respondents wished to remove the separated segment for the evaluation of restorability of the tooth prior to making their final decision. However, this information was not obtained from the survey.

Based on the results of this study, it appears that the presence of a 6-mm periodontal pocket is considered an important factor by most American endodontists when deciding whether to treat the cracked tooth or extract it.

\section{Limitation of the Study}

Our study had a relatively low response rate $(11.28 \%)$ and interpretation of the results should be done with caution. A study conducted by McLeod ${ }^{17}$ used $60 \%$ as a benchmark for responses to surveys. They found a decrease in response to surveys from $61 \%$ in the year 2000 to $36 \%$ in 2008 . Funkhouser et al., ${ }^{18}$ found that the greatest response to surveys is in-person surveys followed by 
paper-based surveys. The lowest response was obtained by online surveys. The reason attributed for a low response rate to online surveys is that people are receiving spam e-mails on regular bases. In addition, older dentists may have a preference for competing paper surveys rather than online surveys.

Another limitation of our study was that we obtained our information anonymously. As a result, even though we obtained data regarding the number of years of experience of the endodontist, we could not corelate that to the answers provided as the survey was anonymous. Hence, no conclusions could be drawn to see if the experience of the clinician changed the treatment approach for the cracked tooth.

\section{References}

1. Cameron CE. Cracked-tooth syndrome. J Am Dent Assoc 1964;68: 405-411.

2. Rivera EM, Walton RE. Cracking the cracked tooth code: detection and treatment of various longitudinal tooth fractures. In: Colleagues for Excellence. Chicago: American Association of Endodontists; 2008.

3. Abbott $\mathrm{P}$, Leow N. Predictable management of cracked teeth with reversible pulpitis. Aust Dent J 2009;54:306-315.

4. Ailor JE Jr. Managing incomplete tooth fractures. J Am Dent Assoc 2000;131:1168-1174.

5. Brynjulfsen A, Fristad I, et al. Incompletely fractured teeth associated with diffuse longstanding orofacial pain: diagnosis and treatment outcome. Int Endod J 2002;35:461-466.

6. Homewood Cl. Cracked tooth syndrome-incidence, clinical findings and treatment. Aust Dent J 1998;43:217-222.

7. Lynch CD, McConnell RJ. The cracked tooth syndrome. J Can Dent Assoc 2002;68:470-475.
8. Berman LH, Kuttler S. Fracture necrosis: diagnosis, prognosis assessment, and treatment recommendations. J Endod 2010;36:442-446. DOI: 10.1016/j.joen.2009.12.018.

9. Eakle WS, Maxwell EH, et al. Fractures of posterior teeth in adults. J Am Dent Assoc 1986;112:215-218.

10. Kang SH, Kim BS, et al. Cracked teeth: distribution, characteristics, and survival after root canal treatment. J Endod 2016;42:557-562. DOI: 10.1016/j.joen.2016.01.014

11. Tan L, Chen NN, et al. Survival of root filled cracked teeth in a tertiary institution. Int Endod J 2006;39:886-889. DOI: 10.1111/j.13652591.2006.01165.x.

12. Sim IG, Lim TS, et al. Decision making for retention of endodontically treated posterior cracked teeth: a 5-year follow-up study. J Endod 2016;42:225-229. DOI: 10.1016/j.joen.2015.11.011.

13. Suksaphar $W$, Banomyong $D$, et al. Survival rates from fracture of endodontically treated premolars restored with full-coverage crowns or direct resin composite restorations: a retrospective study. J Endod 2018;44:233-238. DOI: 10.1016/j.joen.2017.09.013.

14. Krell KV, Caplan D. 12-month success of cracked teeth treated with orthograde root canal treatment. J Endod 2018;44:543-548. DOI: 10.1016/j.joen.2017.12.025.

15. Krell KV, Rivera EM. A six-year evaluation of cracked teeth diagnosed with reversible pulpitis: treatment and prognosis. J Endod 2007;33:1405-1407. DOI: 10.1016/j.joen.2007.08.015.

16. Opdam NJ, Roeters JJ, et al. Seven-year clinical evaluation of painful cracked teeth restored with a direct composite restoration. J Endod 2008;34:808-811. DOI: 10.1016/j.joen.2008.04.011.

17. McLeod CC, Klabunde CN, et al. Health care provider surveys in the United States, 2000-2010: a review. Eval Health Prof 2013;36:106-126. DOI: $10.1177 / 0163278712474001$.

18. FunkhouserE, Fellow JL, etal. Supplementing online surveys with a mailed option to reduce bias and improve response rate: the national dental PBRN. J Pub Health Dent 2014;74:276-282. DOI: 10.1111/jphd.12054. 